\title{
Un Proyecto de Educación Bilingüe Bicultural en el Alto Marañón
}

\author{
José María Guallart*
}

This article describes the secondary boarding school for Aguaruna and Huambisa boys, Instituto Agropecuario Valentín Salegui run by the Jesuit Fathers in the Upper Marañon region. The minimum age for entrance is sixteen, to allow for adequate socialization within their own indigenous culture. Education is bilingual and intercultural. One of the objectives is to form leaders and another is to enable the students to deal with the local Spanish-speaking population with a minimum of culture shock by placing special emphasis on the values of the native culture. Thecurriculum is adapted to de needs of the students and includes training in agriculture and animal raising. The author also provides information about the educational situation among the Aguaruna and Huambisa in the entire region.

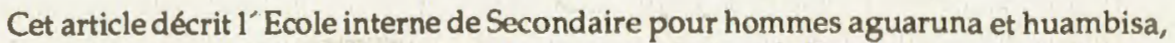
l' Instituto Agropecuario Valentín Salegui, dirigé par les peres jésuites dans la région du Haut-Marañon. L'age minimum d'admission est seiza ans, pour pemettre ainsi una convenable socilisation dans leur culture native. L'éducation est bilingue et interculturelle. Un des objectifs est la formation de leaders et un autre aider l' étudiant a entrer en rapport avec la population hispano-parlanteen souffrant le moindre chocculturel possible, en mettant une emphase spéciale sur les valeurs de la culture native. Le curriculum a étéadapté aux besoins des éleves, et comprend instruction en agriculture et ślcvage d'animaux. L'auteur donne aussi de $l^{\prime}$ information sur la situation éducationelle des aguaruna et huambisa de toute la région. 


\section{Educación bilingüe es:}

- La proporcionada en toda escuela a la que asisten nativos.

- En exclusividad, las regentadas por el Instituto Lingüístico de Verano.

- Las que se tienen para incorporar a la civilización a los salvajes.

- Aquellas en las que se enseña dos lenguas.

- Las erigidas para mantener aislados a los nativos y poder instrumentalizarlo. o manipularlos en determinadas direcciones.

Todas estas respuestas $-\mathrm{y}$ algunas más incompletas, pintorescas o malintencionadas- podrian recogerse si alguien emprendiera una encuesta formal sobre la materia: aunque se ampliara su alcance a sectores no considerados ordinariamente como popula res.

Sin embargo, pese a la desinformación que ha envuelto y todavia envuelve a la educación bilingùe, su ideal -o al menos la palabra que sirve para designarla- ha logrado penetrar ya definitivamente en los ambientes relacionados con la pedagogía. Poco a poco, pero con seguridad, se va abriendo paso a la idea de un sistema educativo que considera necesario que el alumno entienda lo que dice el docente, que el docente entienda al alumno; que ambos colaboren mano a mano para implementar un tipo de formación humana que permita al alumno desenvolverse y progresar en su propio ambiente, sin necesidad de salir de él. Todo esto realizado con el máximo respeto a la persona y sin crear traumas de ningún género.

Con ocasión de la presente nota, he vuelto a leer un trabajo de la doctora Inés Pozzi-Escot, que merecería una divulgación más amplia de la que ha tenido. Me refiero a: Educación Bilingüe en el Perú, una mirada retrospectiva y prospectiva. (mimeografeado, 1975).

A través de sus líneas, todos los que han trabajado con cariffo y dedicación en esta parcela del quehacer pedagógico, podrán reconocer los pasos muy estimables en este campo -en nuestro medio- a partir de la década del cincuenta. La formación de un pequeño pero muy consistente grupo de especialistas nacionales en la Universidad de San Marcos; la creciente claridad de conceptos; la formulación cada vez más ajustada de sus problemas; los intentos todavía no completados de institutos pedagógicos para la formación de maestros bilingües. Pero, sobre todo, el paso de los planteamientos puramente lingứsticos a la problemática global, cultural y socioeconómica implicada: Educación bilingưe, Educación bicultural, Educación pluricultural. Se está llegando a tocar la médula de un gran proyecto nacional peruano como realidad pluricultural. 
Todo esto ha sido logrado en el campo, podríamos decir, conceptual. Pero, iy en la Im.1 Is de la real educación bilingüe? Aunque en este terreno los progresos han sido Inucho más lentos, no han faltado Programas de campo de educación bilingưe, con un al)undante cúmulo de experiencias que todavia faltan evaluar, divulgar y aprovechar.

Uno de estos Programas poco conocidos, es el que vamos a presentar aquí. No por su valur intrínseco (se comenzó y ejecutó con medios técnicos y económicos muy modestos), inu por su valor de estímulo que puede ayudar a otros a emprender programas semefintes, rompiendo la rutina del "hacer lo que se ha hecho siempre".

\section{Antecedentes}

El espacio comprendido entre el pongo de Rentema y el de Mansericha, ha sido tradicionalmente una zona sellada a la penetración de otras lenguas y culturas. Aquî han habitado desde tiempo inmemorial las fracciones nativas de aguarunas y huamThas; pertenecientes ambas a la gran familia jibara. De su real aislamiento-pese a los inevitables contactos con otras etnias- da idea el hecho de que todavía en 1959 el cunso de los no nativos apenas alcanzaba las cien almas.

Poco después de la guerra con el Ecuador (1941), la situación comienza a evolucionar. Aunque hacía años funcionaba en esta zona una diminuta escuela bíblica regentada por un pastor evangélico, sólo en 1947 el Ministerio de Educación abrió en Canampa la primera escuela fiscal para los hijos de un pequeño grupo de colonos. Enseguida los misioneros jesuitas, que acababan de llegar a Niva, recibieron la sollcitud de encargarse de ésta. Recibida, la desdoblaron en dos, abriendo sendos Internados para niños y niñas pertenecientes a la población nativa.

En 1952, el I.L.V. establece en Nazareth su primer centro de alfabetización y comienzan a abrir otros centros por ríos y quebradas, siendo imitados enseguida por los misioneros católicos. Para 1972, cuando la Reforma Educativa de JuanVelascoAlvarado hace asumir vía el Ministerio de Educación todo el sistema escolar, las escuelas suman ya 112, con 228 maestros (casi en su totalidad nativos) y con unos 2,246 alumnos.

Poco antes de 1972, los alumnos egresados de las escuelas de la Misión católica, venían haciendo presión sobre sus maestros:

- ¿Yahora qué? -preguntaban-iQueremos seguir estudiando!

Los misioneros comenzaron a estudiar seriamente el problema pensando abrir en la zona un colegio de secundaria. Para ello contaban con la experiencia invalorable proporcionada por el funcionamiento de sus entonces casi cincuenta escuelas, y el conocimiento del mundo nativo adquirido a través de sus interminables recorridos por trochas y quebradas. 
El proyecto partiría de un objetivo más amplio que el simple problema escolar: Apoyar a la etnia para ayudarla a afrontar el shock producido por una brusca e inevitable discontinuidad cultural, cuyos síntomas se percibían como algo inminente:

- Pérdida del poder de decisión dentro de su mundo, antes prácticamente intacto.

- Quiebra de sus sistemas de control social.

- Inmersión repentina de un nuevo sistema económico

- Aparición de nuevas necesidades reales o simplemente sentidas.

- Inferioridad competitiva ante la población advenediza.

- Ruptura brusca de una incomunicación secular e irrupción masiva de nueva información difícil de descodificar, discernir y asimilar.

- Exhibición, desproporcionadamente prestigiada, de nuevos arquetipos y modelos de vida.

- Exacerbación de las disfunciones culturales que inevitablemente existen en toda cultura.

Ante este desafio, el proyecto presentaba al Ministerio de Educación los siguentes objetivos específicos:

- Valoración del potencial humano nativo, por otra parte plenamente adaptado a su propio medio físico (y por ello elemento imprescindible para toda colonización, pues esta era la consigna de moda en aquel momento), evitando su proletarización y extinción cultural y aún biológica.

- Formación de una élite transformadora en el seno del grupo nativo.

- Preparación para insertar al mismo, sin frustracciones ni traumas, en el contexto del acontecer peruano.

- Considerando que el papel principal de la zona iba a estar a cargo del pequeño agricultor, preparación para asumir este papel con un nivel cultural y productivo lo más alto posible.

- Facilitar al alumnado de las escuelas primarias que aspiren a ello, la oportunidad de una formación que les abra camino a estudios superiores.

Como instrumento, en un contexto regional y humano muy especializado se utilizará la adaptación: Lingüística, cultural y psicológica y la adecuacación también al mercado ocupacional real y a los recursos formativos realmente disponibles.

En un principio, la aprobación se había solicitado sólo para los tres primeros años constituídos en una especie de ciclo básico; se pensaba que era necesaria la contratación de un par de técnicos profesionales - que no se podían encontrar en la zona- para dar el debido peso a la formación profesional de los dos últimos años de la secundaria. Esta aprobación ínicial, fue otorgada por el Ministerio de Educación con fecha 25 de mayo de 1968 y a continuación comenzó a funcionar el programa. Más adelante, cuando se comprobó que no podía contarse con el apoyo de dos ingenieros que inicialmente se habian ofrecido, y ante las expectativas de los alumnos que no podian ser defraudadas, se buscó asesoramiento sólido sobre agricultura, del trópico húmedo de América Latina y sobre la marcha comenzó la preparación del grupo de 
profosores con que ya se contaba. La condición que se juzgó más imprescindible loide un primer momento, fue la de concretar al máximo los contenidos curriculares (to las asignaturas técnicas, para irlos implementando después.

Solicitada la aprobación para completar el segundo ciclo, se obtuvo sin difii ultudes el 25 de abril de 1971. Al año siguiente, pudo egresar la primera promoción del thamante Instituto Parroquial Agropecuario Valentín Salegui, el cual funcionó en la localldad de Chiriaco (1). El Ministerio de Educación pagaría cuatro plazas de profifosionales. De todo lo demás se hacía cargo el Vicariato Apostólico de San Javier. Brama.

Pasaremos ahora a detallar algunas peculiaridades del funcionamiento del pro-

\section{Matrícula}

Solamente para alumnos de lengua y cultura aguaruna selectiva, con un máximo de 25 alumnos por curso y abierta -si es posible- a todas las Comunidades, favoreciendo uspecialmente a las más marginadas geográficamente (por ejemplo las del río Cenepa). Sln ninguna discriminación confesional (normalmente los alumnos católicos constituyen (s) $30 \%)$. Con una edad mínima de 16 años para recibirles plenamente socializados en su propia cultura. Y únicamente para varones; no por razones "morales", sino exclusivamente antropológico-culturales al no poder atender debidamente dos problemas a la vez (2).

Es inútil decir que estos criterios le valieron al programa las acusaciones más diversas: machismo, elitismo (i!), aún la "orden imperativa" de alguno de los jefes de la colonización, que quería imponer la admisión de los hijos de los colonos. Naturalmente su "orden" se desestimó.

La admisión, en régimen de internado era totalmente gratuita. Pronto, sin embargo, ensefó la experiencia que convenía exigir algún tipo de pensión para no hacer "pediguefros" y que lo recibido se estimara en más. (Actualmente la colaboración anual que se pide es el equivalente a uno y medio dólares americanos). La beca incluye comida, alojamiento y atención sanitaria.

(1) El Centro funciona actualmente en Yamakáintsa a unos pocos minutos de bajada en bote, desde la localidad, Imacita, sobre el río Maranón.

(2) El problema de la debida educación de la muchacha aguaruna merecería un tratamiento especial en el que no podriamos entrar aquí. 


\section{El régimen es de internado}

Es la única forma de funcionar con una población dispersa, donde los domicilios de los alumnos pueden distar del centro hasta diez días de viaje. Sin embargo tienen amplia libertad de salir los fines de semana a donde quiera que tengan un familiar o amigo; y la suficiente flexibilidad para adaptarse a los problemas comunitarios o familiares. Pues, aunque no conste "oficialmente", no faltan los alumnos casados sobre todo en los últimos años.

\section{Horarios y calendario escolar}

Las clases son de 45 minutos con un período de $15^{\prime}$ de descanso entre clase y clase, y un recreo de $45^{\circ}$ al terminar la segunda de las cuatro clases matutinas. La tarde se dedica comúnmente a las clases de taller y práctica agropecuaria pues, durante ella el rendimiento de atención disminuye notablemente. El Calendario escolar es el standard de todo el Perú. Unicamente las vacaciones de Fiestas Patrias se prolongan un poco más para los alumnos de 4to. y 5to. que han de realizar en ellas un Trabajo de Práctica en sus Comunidades. Para ello les bastan tres o cuatro días.

Pero, eso sí, existe una seriedad absoluta en el ritmo escolar. Nunca se da el caso de una clase sin profesor. El que falta, justificadamente, ha de dejar trabajo señalado que será supervisạdo por un remplazo o efectuará un intercambio de horas con algún colega. Esto parece absolutamente necesario, pues en caso contrario sobrevendría muy pronto la desmoralización de nuestro alumnado.

Junto a esta máxima seriedad en el trabajo escolar se tienen siempre preparadas actividades novedosas para romper momentos de especial cansancio o de tensiones de cualquier género. Comúnmente al alumno nativo le cuesta, extraordinariamente, el trabajo mental que exija una concentración a la que no está acostumbrado. El cambio triunfa y "se ceba" en una actividad de rendimiento práctico, inmediato y concreto, que exija además habilidad manual en la que él se encuentra como en campo propio.

\section{Infraestructura}

Es muy sencilla y económica. Dieron excelentes resultados los módulos, scparados unos de otros. Cada uno comprende un aula y un dormitorio adjunto para cada curso. Las aulas son lo más abiertas posibles. El piso es de cemento, está cubierta de calaminas (pese a sus inconvenientes por el ruido de la lluvia) y muros reducidos al mínimo; uno no adjuntó al dormitorio y otro para soportar los siempre imprescindibles pizarrones. 
El ajuar escolar inicialmente se improvisó con buenos resultados. Tablas de aserrío de $3 \mathrm{~m}$. de longitud fijas con clavos sobre segmentos de tronco de árbol obtenidos con motosierra y de la altura deseada; unos para los bancos y los otros para los asientos correspondientes. Con el tiempo se substituyeron por carpetas individuales.

\section{La currícula académica}

Se concibió como repartida en dos ciclos: ciclo A (1, 2 y 3 años), destinado sobre todo a suplir los fallos de una primaria hecha en las escuelas de las Comunidades, $y$, ordinariamente, con muy poca consistencia. El nivel, sobre todo en matemáticas y conocimiento del castellano, dejaba y deja mucho que deseár.

En el Ciclo B ( 4 y 5 años), se implementaba subre todo el trabajo de formación verdadera. El alumno está ya mucho más maduro para el esfucrzo. Para ahorro de profesorado calificado, siempre escaso, los alumnos de ambos cursos estudian juntos las mismas asignaturas en ciclos alternos bi-anuales. La deserción escolar por diversas causas aliviaba en este ciclo la carga docente, permitiendo al profesor hacer un verdadero trabajo de artesanía cen cada alumno.

Con algunas modificaciones que indicaremos, el rol de asignaturas es muy parecido al standard de lo que se hacía en los institutos agropecuarios comunes. La originalidad estaba en el tratamiento y contenido de las asignaturas, con una máxima adaptación a la realidad circundante. He aquí los cuadros de asignaturas:

\section{Ciclo A.}

Humanidades

Geografías

Historia del Perú

Lenguaje-segunda Lengua

Educación Cívica

Educación Religiosa
Ciencias

Matemáticas

Botánica

Zoología

Fis. y Ant. Humanas

Fisica y Química

\section{Técnicas}

Dibujo Lineal

Topografía

Agropecuaria

Carpintería

Taller de

Mecánica

Se observará la desaparición de Lengua Extranjera, cuyas horas pasan a Lenguaje de la segunda lengua. La de la Historia Universal que se comprobó enseguida como ininteligible para el alumno en este ciclo; por falta de un horizonte cronológico en su propia cultura y por desconocimiento de la Geografía Universal. Su estudio se trasladó al segundo ciclo. La asignatura Educación Artística, en bloques de horas, se dedicó a Técnicas y Artesanías Nativas bajo la dirección de los "viejos" de las Comunidades contratados ad hoc; esto para ambos ciclos. 


\section{Ciclo B.}

$\begin{array}{lll}\text { Humanidades } & \text { Ciencias } & \text { Cursillos Técricos } \\ \text { Historia Universal } & \text { Matemáticas } & \text { Zoopatología } \\ \text { y de la Zona. } & \text { Física y Química } & \text { Fitopatología } \\ \text { Historia del Perú } & \text { Recursos Naturales } & \text { Ganado Vacuno } \\ \text { Lenguaje-segunda Lengua } & \text { Administración } & \text { Cacao y Arroz } \\ \text { Problemas Socio-Económicos } & & \text { Taller de Mecánica } \\ \text { Educación Religiosa } & \text { Construcciones } \\ & & \text { Rurales } \\ & \text { Topografía } \\ & \text { Enfermería y } \\ & & \text { Primeros Aux. } \\ & \text { Perítajes y } \\ & \text { Tasaciones } \\ & \text { Fruticultura. }\end{array}$

Se notará la supresión de la Economía Política y las asignaturas filosóficas. Aunque no podemos extendernos, notaremos aquí que la Física y Química se estudió alrededor de una aplicación práctica: los suelos. Las Ciencias biológicas alrededor de los Recursos naturales Renovables: silvicultura, piscicultura y fauna silvestre. Los problemas Socio-Económicos de la zona, fueron una iniciación en la investigación social: demografía, censos, utilización de gráficos, estadística elemental, etc. La Educación Religiosa, dado el pluralismo confesional de los alumnos, se enfocó como presentación cutural de la religión oficial del Perú.

Respecto a los "cursillos técnicos", algunos se suprimieron al desaparecer su necesidad (por ejemplo: la enfermería cuando se extendió la presencia de sanitarios especialmente formados en las Comunidades, o el cooperativismo). En otras ocasiones se introdujeron temporalmente nuevas materias, al poder disponerse, para ellas, de algún técnico especialmente calificado. La política general fue la de aprovechar siempre toda posibilidad formativa accesible. Por ejemplo, actualmente, los cursos obligatorios de Reparación y Mantenimiento de motores de "fuera de borda" de utilidad insustituible en la zona.

También funcionaron, y funcionan extra-curricularmente, la administración del bazar escolar, mecanografía, impresiones a mimeógrafo, etc.

\section{Valores y actitudes}

En un Proyecto de educación bicultural, se consideró de capital importancia rebasar el campo de los conocimientos y técnicas, y pasar al de la creación de las actitudes necesarias en una nueva situación junto con la conservación de valores poseídos pero en 
peligro y/o, la introducción de otros nuevos. Esto no puede reflejarse en un cuadro de asignaturas, pero debe entrar en todas ellas y aparecer, sobre todo, en las propias actitudes del personal docente y directivo.

Lo que aquí se procuró inculcar con todo ahinco fue:

- Sentido de responsabilidad hacia el propio grupo combatiendo el egoismo insular de familia o de clan.

- Estima de la propia identidad cultural.

- Conocimiento crítico y evaluativo de la propia cultura.

- Idem de la nueva, con sus ventajas y disfunciones.

- Espíritu de reflexión atenta y crítica sobre las nuevas situaciones.

- Integración positiva y optimista al resto de la sociedad.

- Conocimicnto y asimilación de la importancia de derechos y deberes con la capacitación necesaria para ejercerlos.

- Valor del trabajo en común, hondamente arraigado en la cultura jíbara, pero ampliando sus límites a la cooperación con toda la etnia, etc...

Todo este programa curricular parecerá demasiado ambicioso y monolítico. Su continuidad integra a través de más de 20 años y fue, casi imposible de mantener. Lo que se ha expuesto corresponde estrictamente al periodo transcurrido entre la fundación y 1980. Pero en líneas generales se ha conservado substancialmente y, aún, enriquecido. Aunque no ha dejado de notarse el efecto "corsé-ortopédico-uniformador" de los planes y programas del Ministerio de Educación que ha hecho derivar un poco la currícula de los Planes comunes a todos los Centros Agropecuarios del Perú. Tal ha sucedido, por ejemplo con la reintroducción de la Historia Universal en el primer año, o la supresión de la asignatura de Problemas. Socio-Económicos de la zona. Pero en líneas generales la orientación ha sido substancialmente la misma.

\section{El profesorado}

Se consideró imprescindible la presencia de un núcleo, aunque reducido, sí de buena calidad y capacitación pedagógica, en forma de equipo muy integrado. Junto a ellos la presencia de profesores nativos -todos ex-alumnos, ha sido el complemento enriquecedor. En ellos, se consideró de máxima importáncia más el sentido común para adaptarse al alumno que la exhibición presuntuosa de conocimientos... con frecuencia poco asimilados. El profesorado se ha ido formando en el propio Centro, junto al cuidadoso seguimiento de parte del profesor principal de la correspondiente línea. Así, por ejemplo, pudo formarse un magnífico profesor de matemáticas nativo que hoy ocupa un puesto de alta responsabilidad en una de las oficinas de eduación de zona. Un respaldo pedagógico continuo de los profesores junto con un "syllabus" de las asignaturas bien pensado y concreto, pueden ser los firmes pilares de una buena educación bilingüe con profesores nativos. 


\section{Los textos}

El Proyecto tropezó, desde el principio, con la falta de textos adecuados a los reales conocimientos lingüísticos del alumno. Tanto el vocabulario, como la ambientación cultural de los existentes exigen un trabajo paralelo que retrasa innecesariamente todo el progreso en los conocimientos base. Por otra parte hay que reconocer que, de existir, serian una fuente de enriquecimiento invalorable para el dominio del castellano. Precisamente para esta última asignatura tan capital, la falta de textos aptos ha constituido con frecuencia un factor limitante. El editarlos resulta antieconómico, además si se trata de pequeños números. En la mayoría de las asignaturas, en cambio, dio buen resultrado el sistema de "fichas-resumen", explicadas por el profesor y copiadas después por el alumno. Copiadas se entiende, no en la clase ni del pizarrón, ni menos dictadas según la costumbre tan divulgada y tan esterilizante.

De manera un poco sistemática, se ha venido preparando a lo largo del tiempo un repertorio de dichas fichas resumen. Existe ya el curso entero de matemáticas; de Recursos Naturales Renovables, Suelos, Fito y Zoo-patología, Frutales, Vacunos, Porcinos, Aves, Topografía, Dibujo lineal, Anatomia Humana. Parcialmente de Física y Química y, últimamente, de Historia de la Zona y Geografía. En lo que se ha tenido más atraso por su especial dificultad, ha sido en lenguaje, quizá la asignatura mas importante, la segunda lengua. Aunque en esto se está trabajando metódicamente.

Por no extendernos aquí en forma desmesurada, omitimos hablar del sistema de evaluación, tan dificultoso de establecer para un alumno que no domina nuestra lengua ni nuestros sistemas de expresión.

Incidentalmente notaremos que, sobre todo últimamente, hemos visto la utilidad que tendría introducir dos nuevas asignaturas. Una de ellas sería una especie de Cosmologia-Antropología. El alumno jibaro esucha ya muchas cosas que le introducen en un mundo nuevo y sus ideas tradicionales sobre ellas son sumamente elementales, y de tipo exclusivamente mágico. Habría que prepararle y ayudarle a aclarar sus ideas sobre ello.

Otra sería una asignatura que aborda y expresa directamente la manera de estudiar, escuchar, resumir, responder que, ulteriormente, lo capacitará para la asistencia a los centros de enseñanza de tipo Superior, para los que ahora se encuentra en condiciones de inferioridad.

\section{Evaluación del proyecto}

Existe un verdadero peligro, aparecer demasiado apologékicos o triunfalistas. El Proyecto necesitaría una evaluación detenida en la que, sin duda, habrian de aparecer abundantes fallas. Muchas de ellas las perciben intuitivamente los promotores.

Sin embargo, no creo que el conjunto quite al Proyecto su principal valor, que me parece el esfuerzo innovador y el de estímulo para que se pueda animar a otros a 
emprender nuevas exploraciones en el enfoque de la educación bilingüe-bicultural. De todas formas consignaremos aquí unos cuantos datos esquemáticos significativos.

Continuidad. Veintiún años de funcionamiento. Diecisiete promociones egresadas. Por el proyecto han pasado cuatro directores pero dos de ellos desemperaron su cargo por ocho y nueve años consecutivos, respetivamente, lo que aseguró la suficiente coherencia decriterios.

Matrícula. En su matrícula han figurado 753 alumnos. Por su más larga permanencia en el Colegio pudieron recibir plenamente su impronta 496. Egresaron concluidos sus estudios (hasta 1988) 182.

Procedieron de 130 Comunidades distintas.

Deserción escolar total: Permanecieron relativamente poco tiempo $34 \%$

De estos culminaron sus estudios el $36.69 \%$

Causas de deserción escolar: (deserción total 51.18\%).

Matrimonio y problemas familiares $33 \%$.

Servicio Militar Obligatorio 33\%. Desánimo, diversus $33 \%$

\section{Colocación al egresar: $\quad 68.8 \%$ magisterio (todos quedaron en la zona). \\ $17 \%$ agricultura en sus comunidades \\ $4.6 \%$ Diversos empleos con sueldo \\ $3.31 \%$ liderazgo en sus Comunidades, organizaciones.}

Para satisfacción de los promotores, sólo uno de los egresados salió a trabajar fuera de la zona.

En la introducción de este rabajo, quisimos reseñar los avances logrados entre nosotros respecto a la educación bilingüe durante las últimas décadas: sus avances y sus carencias. Ahora quizá sea oportuno cerrarlo con algunos datos sugestivos de lo que falta por hacer en ese campo.

Seria presunción intentar hacerlo abarcando toda la problemałtica educacional de la totalidad de las etnias de nuestra Amazonia peruana. Cada una de ellas constituye un caso tan particular como son sus respectivas coyunturas de contacto con la sociedad nacional, etc. Por eso me voy a referir únicamente a un caso concreto conocido por referencias directas: el de la etnia jíbara que se extiende por el distrito de Imaza, Condorcanqui, cabeceras del río Mayo y provincia de Alto Amazonas en Loreto.

Las escuelas primarias a las que asisten ahumnos pertenecientes a dicha etnia, y que como se recordará eran 112 con 2,246 matriculados en 1972; son actualmente 116, proporcionando escolaridad a 10,159 muchachos nativos. Y a estos habría que afladir los que asisten a otros centros mezclados con los castellano hablantes, hijos de colonos o riberef́os. Esto podrá proporcionarnos los datos para una proyeccción de lo que debería ser la demanda de matrícula para secundaria. 
El estado actual de las estructuras educativas para la población de cultura jibara queda presentado en los siguientes cuadros; uno referente a la educación primaria que puede proporcionarnos la base para un cálculo de potencial demanda educativa para secundaria y otro para la propia educación de este último grado.

Es interesante notar que no es posible encontrar, consolidados, los datos estadísticos pertinentes. Al parecer estos ni existen en la dírección central de Educación Bilingüe del Ministerio. Los aquí recogidos se colectaron directamente en los Centros y cuando esto no fue posible, se establecieron proyecciones a base de años anteriores partiendo de una interpretación "minimista". En este último caso se indica el hecho con un asterisco, o con un signo de interrogación.

Etnia jíbara. Estadísticas oducacionales pạa 1989.

\section{EDUCACION PRIMARIA}

\begin{tabular}{|c|c|c|c|c|}
\hline \multirow[b]{2}{*}{ Supervisión o USE } & \multirow[b]{2}{*}{ Centros } & \multirow[b]{2}{*}{ Alumnos } & \multicolumn{2}{|c|}{ Docentes } \\
\hline & & & No nativos & nativos \\
\hline Nieva & 130 & 6.379 & & 215 \\
\hline Imacita & 65 & 3.385 & & 133 \\
\hline Moyobamba & 13 & $399 ?$ & & $21 ?$ \\
\hline San Lorenzo & 22 & $880 ?$ & & $24 ?$ \\
\hline \multicolumn{5}{|c|}{ EDUCACION SECUNDARIA } \\
\hline \multirow[t]{7}{*}{ Nieva } & Nieva & 95 & 10 & .1 \\
\hline & Olaya & 7 & - & \\
\hline & Kigkís & 60 & 2 & 1 \\
\hline & Ciro Alegría & 20 & 4 & \\
\hline & Pto. Galilea & 40 & 5 & 1 \\
\hline & Yutupis & 30 & $?$ & \\
\hline & Huampaní & 61 & 7 & \\
\hline \multirow[t]{7}{*}{ Imacita } & Acosta Herrera & a 105 & 12 & 1 \\
\hline & Sushunga & 27 & 1 & 1 \\
\hline & Nazareth & 79 & - & 2 \\
\hline & Chiriaco & 65 & - & 9 \\
\hline & Túpac Amaru & 18 & - & \\
\hline & Chipe & 62 & 3 & 2 \\
\hline & Yamakayentsa & 140 & 4 & 4 \\
\hline \multirow[t]{6}{*}{ San Lorenzo } & San Lorenzo & 70 & 11 & \\
\hline & Siramirisa & 6 & 3 & \\
\hline & Ushpayacu & 26 & ? & \\
\hline & Barranquita & 40 & 3 & \\
\hline & Morona & 23 & 2 & \\
\hline & Apaga & 18 & $?$ & \\
\hline Rioja & Bajo Naranjillo & 80 & 2 & 4 \\
\hline
\end{tabular}

N.B. En los ser̃alados con un guión estudian mezclados castellano hablantes y nativos. 
En Huampani e Imacita existen sendos Colegios de Secundaria para adultos cuyo profesorado es el mismo que el del Colegio convencional.

La situación en estos centros es la siguiente:

El $75.5 \%$ de sus docentes ignora la lengua nativa; no digamos su cultura y lo que habrán de ser sus expectativas para cuando terminen sus estudios. Para agravar esta situación, la mayoria de estos profesores no nativos permanccen en sus plazas solamente el tiempo suficiente para adquirir derechos de escalafón y pedir enseguida -al año- su traslado a otra plaza menos sacrificada para ellos. $Y$ así no pueden ni siquicra enriquecerse con la experiencia proporcionada por una mayor convivencia.

Ni qué decir tiene que los planes y programas son los establecidos por el Ministerio de Eaucación para todo el Perú (Léase la Costa y Lima), por cierto que en alguno de ellos he visto que ¡se enseña hasta inglés! el profesor.

Y no hablemos de textos y otros subsidios adaptados que, a veces, ni existen para

Esta es la cruda realidad de una situación. Estamos todavía muy lejos de aquel generoso -y un poco optimista-cronograma para le implementación de la Educación bilingüe que apareció en 1972 en el folleto "Política Nacional de Educación Bilingüe" del Ministerio de Educación. Y aquello era para la Educación Primaria y en la Secundaria, parece que todavia ni se piensa.

Si existe un verdadero propósito de hacer posible la educación a todos los niveles, para todos los peruanos aún para los más pobres y marginados, creo que algo se podría hacer. No se trata de gastos cuantiosos, más calamina y cemento para infraestructuras, ni multiplicación indefinida de nuevas plazas docentes. La consigna sería aprovechar integramente lo que ya se tiene y potenciarlo con una ayuda que no sería muy costosa. Ahí tenemos por ejemplo al profesorado nativo. Con sus modestos reciclajes pedagógicos (y ojalá fueran estos siempre, bien adaptados a ellos), y sobre todo con la riqueza de su entusiasmo por ayudar a la propia gente, podrían dar, con la debida orientación, insospechado rendimiento. Esta ayuda se está dando ya de parte de algunas instituciones particulares. Pero ipor favor!, no nos quedemos tranquilos esperando ver surgir también una "pedagogía informal". O si se hace esto, lléguese al menos a las últimas consecuencias. 